\title{
SPECTROSCOPIC OBSERVATIONS OF VISUAL DOUBLE STARS
}

\author{
FREDERICK R. WEST \\ Geosciences Dept., State Univ. College at Buffalo, Buffalo, N.Y., U.S.A.
}

There are certain visual double stars which, when close to a node of their relative orbit, should have enough radial velocity difference $(10-20 \mathrm{~km} / \mathrm{s})$ that the spectra of the two component stars will appear resolved on high-dispersion spectrograms $(5 \AA / \mathrm{mm}$ or less) obtainable by use of modern coudé and solar spectrographs on bright stars. Both star images are then recorded simultaneously on the spectrograph slit, so that two stellar components will appear on each spectrogram.

The method essentially treats the visual binary star as a spectroscopic binary, but uses at least the elements $a^{\prime \prime}$ and $i$ of the visual orbit which are unobtainable from the radial velocity curve. If the apparent visual binary orbit is accurately known the elements $P, \omega, e$ and $T$ can be used along with the radial velocity difference $V_{r A}-V_{r B}$ between the $A$ and $B$ components (considering a two-body system) to find both the total mass $\left(\mathfrak{M}_{A}+\mathfrak{M}_{B}\right)$ and the absolute parallax $\pi^{\prime \prime}$ of the system. If $V_{r A}-V_{r B}$ is given in $\mathrm{km} / \mathrm{s}$, then

$$
\begin{aligned}
\left(\mathfrak{M}_{A}+\mathfrak{M}_{B}\right) & =P\left(1-e^{2}\right)^{3 / 2}\left[\begin{array}{c}
\left|V_{r A}-V_{r B}\right| \\
29.76 \sin i[e \cos \omega+\cos (v+\omega)]
\end{array}\right]^{3}, \\
\pi^{\prime \prime} & =\begin{array}{l}
29.76 a^{\prime \prime} \sin i \\
\left|V_{r A}-V_{r B}\right| P
\end{array}\left(1-e^{2}\right)^{-1 / 2}[e \cos \omega+\cos (v+\omega)],
\end{aligned}
$$

where $\left(\mathfrak{M}_{A}+\mathfrak{M}_{B}\right)$ is given in solar mass units and $P$ in sidereal years. The above method for finding stellar masses and parallaxes was first pointed out by See, and used by Wright and Hussey to get independent values of $\pi^{\prime \prime}$ for $\alpha$ Centauri and $\delta$ Equulei, respectively (Aitken, 1964). Recent work on $\delta$ Eql will be discussed below.

This method can be used successfully only on visual binary stars that fulfill certain conditions. Six of these conditions are:

(1) The combined magnitude of the binary must be brighter than $m_{v}=8^{m}$.

(2) The components must be of nearly equal brightness and spectral type; $\Delta m_{v} \leqslant 0$. 5 .

(3) The period of revolution must be short $(P \leqslant 60 \mathrm{yr})$.

(4) The inclination of the orbit plane must be high $\left(60^{\circ}<i<120^{\circ}\right)$.

(5) The spectral lines must be narrow for each component. This eliminates almost all stars of spectral classes $\mathrm{O}, \mathrm{B}$, and $\mathrm{A}$ as well as rapidly rotating stars.

(6) There must be not too many overlapping lines and molecular bands in the spectrum of each component. This probably eliminates stars of spectral class $\mathrm{M}$.

The above conditions drastically reduce the number of visual binary stars whose spectra can be resolved spectroscopically. Of the 600 visual binary stars for which orbits have been determined, perhaps at most, 10-15 can be successfully resolved. An unpublished computer survey of Worley's orbit catalogue (1963) carried out by M. Dworetsky of the Astronomical Department at the University of California 
at Los Angeles showed that, besides $\delta$ Eql, the spectra of only one or two additional visual binaries could be resolved by this method when they pass through their nodes between the years 1985 and 2000 .

If the visual orbit is accurately determined the total mass and the parallax can be computed from (1) and (2), respectively, if a reliable radial velocity difference can be obtained. For many close visual binaries where the angular separation is always less than 0.5 , the orbital elements may be considerably uncertain. Reliable radial velocities can be used to improve the values of $P, e, \omega$, and $T$ found from the visual orbit.

In determining the individual masses from the total system mass it is generally best to use the astrometric mass ratio (fractional mass), viz.

$$
B=\mathfrak{M}_{B} /\left(\mathfrak{M}_{A}+\mathfrak{M}_{B}\right)
$$

when it is available, since $B$ can usually be found to a much greater accuracy $( \pm 0.01$ p.e.) than the mass ratio derived from the ratio of the amplitudes $K$ of the radial velocity curves,

$$
\mathfrak{M}_{B} / \mathfrak{M}_{A}=K_{A} / K_{B}
$$

The accuracy of this ratio usually is near 0.05 p.e. at $K \simeq 10 \mathrm{~km} / \mathrm{s}$.

Research on the visual binary stars for which resolution of the component spectra is likely seems to have been, until recently, the study by individual astronomers of stars of special interest. We just heard Dr. Batten's description of his work on the triple system ADS 8189, and on $\delta$ Eql and $\Sigma$ 2173. Dworetsky (1967) has also obtained spectrograms of $\delta \mathrm{Eql}$, at $4 \AA / \mathrm{mm}$ dispersion. The results of his measurements of $V_{r A}-V_{r B}$ for that star seem to be inconsistent with the visual orbit elements. The present author noted that $\Sigma 2173$, spectral type G5 to G8, would pass through a node early in 1965 . Two spectrograms of $8.9 \AA / \mathrm{mm}$ dispersion were obtained at Kitt Peak National Observatory in June 1965; there was some evidence of marginal resolution (West, 1966). These questionable results induced Batten and Fletcher to obtain spectrograms of $\Sigma 2173$ at higher dispersion. The plates they obtained in 1968 and 1969 at $2.5 \AA / \mathrm{mm}$ show the two component spectra clearly resolved. A joint paper on the work on $\Sigma 2173$ by Batten, Fletcher, and West is now in press.

The author has also obtained spectrograms of ADS 14893: one in June 1965, three in November 1968, and fifteen in July 1969. ADS 14893 is a triple system. The visual orbit gives $P=12.2 \mathrm{yr}, a^{\prime \prime}=0$." 18 ; both visible stars are of spectral type F5 to F8. One of them is a single-line spectroscopic binary with $K \simeq 75 \mathrm{~km} / \mathrm{s}$, and $P \simeq 2.2$ days. A complete radial velocity curve cannot yet be drawn, so the other orbital elements and the mass function are still unknown. To obtain the total mass and parallax of such a triple system is considerably more complex than the above treatment of a two-star system. Abt and Kallarakal (1963) have dealt with a similar system, viz. 1 Geminorum.

Photoelectric observations of ADS 14893 were carried out on 2 nights in August 1969 by Kwan-Yu Chen and the author, at the University of Florida, to search for a possible eclipse, but none was found.

The visual orbit of $\Sigma 2173$ is quite accurately known, and the radial velocity curve 
will probably not change the orbital elements much, if any. Hence, the mass and parallax can be directly computed from (1) and (2). The small value of $a^{\prime \prime}$ for ADS 14893 indicates that its visual orbit is probably uncertain. Long-term spectroscopic observations of this system may well lead to greatly changed and improved values of the elements $P, e, \omega$ and $T$ before any reliable mass and parallax determinations can be made.

\section{References}

Abt, H. A. and Kallarakal, V. V.: 1963, Astrophys. J. 138, 140.

Aitken, R. G.: 1964, The Binary Stars, 2nd rev. ed., Dover Publ., New York, pp. 224-225.

Dworetsky, M.: 1967, Astron. J. 72, 297.

West, F. R.: 1966, Astron. J. 71, 186.

Worley, C. E.: 1963, A Catalog of Visual Binary Orbits, Publ. U.S. Naval Obs., 2nd series, Vol. 18, pt. III.

\section{Discussion}

Franz: With the image tube spectrographs now available, is it still necessary to limit so stringently the magnitude of the stars that can be observed?

West: Possibly not. I have not used the image tubes at the solar telescope at Kitt Peak because of the large image distortion.

Franz: Distortion should not be serious where the separation of lines is concerned, and not the position of the lines with respect to wave lengths.

Heintz: The magnitude limitation is not serious since we do not have many good orbits for faint stars.

Franz: We might get them just by help of radial velocities.

Strand: It is highly important that a sufficient number of standard stars are observed since the radial velocities are obtained with long intervals in between the observing series. 